\title{
Commentary: underutilization of curative-intent therapy for patients with muscle-invasive bladder cancer in Sweden mimics the United States
}

\author{
Timothy N. Clinton, Michal Wiseman, Aleksandra Walasek, Eugene Pietzak \\ Department of Surgery, Urology Service, Memorial Sloan Kettering Cancer Center, New York, NY, USA \\ Correspondence to: Eugene Pietzak. 1275 York Ave., New York, NY 10065, USA. Email: pietzake@mskcc.org. \\ Provenance: This is an invited article commissioned by the Section Editor Dr. Xiao Li (Department of Urology, Jiangsu Cancer Hospital \& Jiangsu \\ Institute of Cancer Research \& Affiliated Cancer Hospital of Nanjing Medical University, Nanjing, China). \\ Comment on: Westergren DO, Gårdmark T, Lindhagen L, et al. A Nationwide, Population Based Analysis of Patients with Organ Confined, Muscle \\ Invasive Bladder Cancer Not Receiving Curative Intent Therapy in Sweden from 1997 to 2014. J Urol 2019;202:905-12.
}

Submitted Nov 27, 2019. Accepted for publication Dec 17, 2019.

doi: $10.21037 /$ tau.2019.12.22

View this article at: http://dx.doi.org/10.21037/tau.2019.12.22

\section{Introduction}

Patients with muscle-invasive bladder cancer (MIBC) have a narrow window of cure that requires aggressive treatment in order to attempt to achieve cure. Guidelines recommend the use of curative intent therapy (CIT) involving radical cystectomy (RC) with pelvic lymph node dissection and neoadjuvant chemotherapy for those eligible for cisplatin. Alternative treatments, such as partial cystectomy or chemoradiation, are indicated for patients who are medically unfit for RC, for patients who decline RC, or in select patients with favorable tumors who desire bladder preservation $(1,2)$.

\section{CIT in Sweden}

Despite the recommendations of guidelines, multiple population-level studies have demonstrated that RC is underused as CIT for patients with MIBC. A recently published study used a large nationwide database in Sweden (BladderBase) to describe the disease progression and survival of patients with MIBC who did not receive CIT, which has largely been undefined (3). CIT was defined as radiotherapy or RC with or without neoadjuvant chemotherapy (trimodal therapy was not used). Of the 9,811 patients with cT2-T4 disease, 5,592 (57\%) did not receive CIT. Within the cohort, 1,352 patients had "organconfined" disease (T2-3M0), and these patients had a median overall survival of 11 months. The study reported a median of 2.4 hospitalizations (determined by hospital billing information) in the year after diagnosis in these patients. Approximately half of this subgroup had known cN0 stage, and the median overall survival of these patients was only 2 months longer as compared to the remainder of patients with "organ-confined" disease. Although this population-level database has limited granularity in its data, the study demonstrates that majority of patients with MIBC are not undergoing CIT and that the survival of these patients is poor.

The study found that those who did not undergo CIT were generally older, had a higher comorbidity score, had a lower level of education, and were more likely to be widowed. Patients who did not undergo CIT experienced substantial disease-specific morbidity, poor overall survival, and increased hospitalizations, specifically in the year following diagnosis. Interestingly, the number of patients who did not receive CIT decreased over time. Interpretation of this study is difficult because BladderBaSe does not capture the reasons a patient did not receive CIT. It is possible that the reasons were physician-determined, such as age, comorbidity, or performance status, but they could have also been patient-determined, such as patient refusal.

A similar study using a Swedish database within Stockholm County sought to determine the natural history of untreated MIBC (4). One hundred twenty-six patients 
with cT2-4NanyM0 MIBC were evaluated, with 62 (49\%) receiving CIT of either radiation therapy or RC. It is important to note that this study evaluated patients between 1995 and 1996, so those patients receiving CIT did not benefit from neoadjuvant chemotherapy or refined clinical care pathways for enhanced recovery. This study found that those with an older age and higher T-stage were less likely to receive CIT. The 5-year cumulative incidence of cancerspecific mortality was $86 \%$ in patients who did not undergo CIT and $48 \%$ in those who did receive treatment $(\mathrm{P}<0.001)$. This study did not have the necessary data to compare the number of hospitalizations after diagnosis. Collectively, these studies suggest that CIT is being underused in Sweden.

\section{CIT in the United States}

In the United States, there are large databases that have been used to evaluate the use of CIT in patients with MIBC. The National Cancer Database (NCDB) was queried for patients with stage II-IV MIBC (excluding patients with pT4b disease or with distant metastases) from 2004 to 2008 (5). This work demonstrated that 37\% of eligible patients did not receive standard of care CIT, defined as either radical or partial cystectomy or definitive chemoradiation. When stratified for age, $65 \%$ of those $81-90$ years old and $85 \%$ of those over 90 years old did not receive CIT. Further, only $13 \%$ of eligible patients over 80 years old underwent RC (6). However, the study reported that institutions with the highest volume of patients had increased use of CIT and that there was a significant increase in the use of CIT in National Cancer Institute-designated centers.

Two studies have used Surveillance, Epidemiology, and End Results (SEER)-Medicare data to assess the rate at which those with cT2-3 disease undergo RC with curative intent $(7,8)$. These data suggest that CIT is underused significantly in the United States, with only $18-21 \%$ of eligible patients (based on stage) undergoing RC. Although this rate is much lower than that reported in the Swedish cohorts, the BladderBase and Stockholm County studies included patients also undergoing radiotherapy. Further evaluation of SEER data found that RC was less likely in those of older age, black race, and increased comorbidity score and in areas with decreased urologist density (7).

\section{Implications and importance}

The underutilization of CIT in both the United States and Sweden is multifaceted, and there are notable differences between the two countries. Sweden has universal health coverage, so access to care and socioeconomic issues do not tend to play a role in receiving or electing to receive CIT. In the United States, it has been shown that those uninsured or on Medicaid were less likely to receive CIT $(5,9)$. SEERMedicare data have also demonstrated that access to care, especially for rural populations who may need to travel more than 50 miles to the nearest urologist, is yet another limitation to undergoing RC $(8,10)$.

Multiple retrospective institutional databases have shown no significant difference in morbidity or cancerspecific survival between older and younger patients $(11,12)$. It is clear is that morbidity and mortality from RC is associated with performance status and comorbidities but not age. Some of the temporal trends observed in the BladderBaSe study may reflect the increased willingness of physicians to use $\mathrm{RC}$ in octogenarians. As robotic RC has been demonstrated to be noninferior to open approaches, it is possible that the ability to perform RC robotically is contributing to its increased use, even in elderly patients (13-15). Due to the complex nature of caring for elderly, comorbid patients, it is possible that centralization of care for patients with bladder cancer, which is employed in some European countries, may be warranted. Future work focused on identifying a validated risk assessment to guide clinical decisions is needed (16).

Of course, there are some patients who are truly not eligible for radical surgery or those who electively refuse surgery for curative intent. Bladder-preservation strategies, including trimodal therapy, have a 5 -year cancer-specific survival of $50-82 \%$ in select patients $(17,18)$. For those patients who choose bladder preservation or who are unfit for RC, trimodal therapy with maximum transurethral resection of bladder tumor, appropriately dosed radiation, and radiosensitizing chemotherapy should be used as CIT. Unfortunately, it is clear from the low use in both Sweden and the United States that this alternative is not pursued. These studies have demonstrated that survival is less than a year without the use of any therapy, and thus it is extremely important that all patients, regardless of age or comorbidities, are informed of all treatment options. 


\section{Summary}

The Swedish BladderBase study provides further evidence that the underutilization of CIT for MIBC is a worldwide issue. This study reaffirms that those who are not receiving CIT tend to be elderly, increased comorbidities, less educated and widowed so the support at home is limited. As urologists, it is important to identify that those patients requiring more complex care who are likely to be better served at high volume cancer centers. While the increased use of robotic surgery and enhanced recovery pathways has allowed for more patients to be eligible for $\mathrm{RC}$, it is vital to engage multidisciplinary care to achieve the best outcomes. By incorporating a multidisciplinary team, those patients who are deemed ineligible for RC or electively refuse surgery should be offered trimodal therapy as attempt to provide CIT. Through engagement of multiple specialists, the continued trend of underutilization of CIT in the United States and Sweden must improve for the survival and care of bladder cancer patients.

\section{Acknowledgments}

Funding: This work was supported in part by a grant from the NIH/NCI to Memorial Sloan Kettering Cancer Center (P30 CA008748), Sidney Kimmel Center for Prostate and Urologic Cancers, NIH/HCATS Grant UL1-TR002384 and by SPORE in Bladder Cancer P50 CA221745.

\section{Footnote}

Conflicts of Interest. The authors have no conflicts of interest to declare.

Ethical Statement. The authors are accountable for all aspects of the work in ensuring that questions related to the accuracy or integrity of any part of the work are appropriately investigated and resolved.

\section{References}

1. Flaig T, Spiess P, Agarwal N, et al. NCCN Clinical Practice Guidelines in Oncology: Bladder Cancer Version 4.2019. In: National Comprehensive Cancer Network. Available online: https://www.nccn.org/ professionals/physician_gls/pdf/bladder.pdf. Accessed November 26, 2019.

2. Chang SS, Boorjian SA, Chou R, et al. Diagnosis and treatment of non-muscle invasive bladder cancer: AUA/ SUO guideline. J Urol 2016;196:1021-9.

3. Westergren D, Gardmark T, Lindhagen L, et al. A nationwide population based analysis of patients with organ confined, muscle invasive bladder cancer not receiving curative intent therapy in Sweden from 19972014. J Urol 2019;202:905-12.

4. Martini A, Sfakianos J, Renstrom-Koskela L, et al. The natural history of untreated muscle-invasive bladder cancer. BJU Int 2019. [Epub ahead of print].

5. Gray PJ, Fedewa SA, Shipley WU, et al. Use of potentially curative therapies for muscle-invasive bladder cancer in the United States: results from the National Cancer Data Base. Eur Urol 2013;63:823-9.

6. Fischer-Valuck BW, Rao YJ, Rudra S, et al. Treatment patterns and overall survival outcomes of octogenarians with muscle invasive cancer of the bladder: an analysis of the National Cancer Database. J Urol 2018;199:416-23.

7. Williams SB, Huo J, Chamie K, et al. Underutilization of radical cystectomy among patients diagnosed with clinical stage T2 muscle-invasive bladder cancer. Eur Urol Focus 2017;3:258-64.

8. Gore JL, Litwin MS, Lai J, et al. Use of radical cystectomy for patients with invasive bladder cancer. J Natl Cancer Inst 2010;102:802-11.

9. Fedeli U, Fedewa SA, Ward EM. Treatment of muscle invasive invasive bladder cancer: evidence from the National Cancer Database 2003 to 2007. J Urol 2011;185:72-8.

10. Mossanen M, Izard J, Wright JL, et al. Identification of underserved areas for urologic cancer care. Cancer 2014;120:1565-71.

11. Donat SM, Siegrist T, Cronin A, et al. Radical cystectomy in octogenarians - does morbidity outweigh the potential survival benefits? J Urol 2010;183:2171-7.

12. Clark PE, Stein JP, Groshen S, et al. Radical cystectomy in the elderly. Cancer 2005;104:36-43.

13. Bochner BH, Dalbagni G, Marzouk KH, et al. Randomized trial comparing open radical cystectomy and robot-assisted laparoscopic radical cystectomy: oncologic outcomes. Eur Urol 2018;74:465-71.

14. Parekh DJ, Reis I, Castle EP, et al. Robot-assisted radical cystectomy versus open radical cystectomy in patients with bladder cancer (RAZOR): an open-label, randomised, phase 3, non-inferiority trial. Lancet 2018;391:2525-36.

15. Matulewicz RS, DeLancey JO, Manjunath A, et al. National comparison of oncologic quality indicators between open and robotic-assisted radical cystectomy. Urol Oncol 2016;34:431.e9-15. 
16. Woldu SL, Sanli O, Clinton TN, et al. Validating the predictors of outcomes after radical cystectomy for bladder cancer. Cancer 2019;125:223-31.

17. Kulkarni GS, Hermanns T, Wei Y, et al. Propensity score analysis of radical cystectomy versus bladder-sparing trimodal therapy in the setting of a multidisciplinary

Cite this article as: Clinton TN, Wiseman M, Walasek A, Pietzak E. Commentary: underutilization of curative-intent therapy for patients with muscle-invasive bladder cancer in Sweden mimics the United States. Transl Androl Urol 2019;8(Suppl 5):S542-S545. doi: 10.21037/tau.2019.12.22 bladder cancer clinic. J Clin Oncol 2017;35:2299-305.

18. Ploussard G, Daneshmand S, Efstathiou JA, et al. Critical analysis of bladder sparing with trimodal therapy in muscle-invasive bladder cancer: a systematic review. Eur Urol 2014;66:120-37. 